\title{
VALOR NUTRICIONAL Y CARACTERIZACIÓN DE LOS ÁCIDOS GRASOS DEL \\ CHONTACURO Rhynchophorus palmarum $L$.
}

\section{THE NUTRITIONAL VALUE AND CHARACTERIZATION OF THE FATTY ACIDS OF CHONTACURO Rhynchophorus palmarum $L$.}

\author{
Ana Espinosa M., ${ }^{1}$ Ana Hidalgo A., ${ }^{1}$ Eduardo Mayorga LI. ${ }^{1}$ \\ Recibido: 30 de septiembre 2019 / Aceptado: 10 de diciembre 2019 \\ DOI: 10.26807/ia.v8i1.122
}

Palabras clave: Chontacuros, parámetros fisicoquímicos, Rhynchophorus palmarum, valor nutricional Keywords: Chontacuros, physicochemical parameters, Rhynchophorus palmarum, nutritional value

\section{RESUMEN}

La presente investigación tuvo como finalidad determinar el valor nutricional, así como identificar los tipos de ácidos grasos que componen la grasa de los chontacuros de la especie Rhynchophorus palmarum L. Estos fueron recolectados en los mercados de la ciudad del Puyo de la provincia de Pastaza, siendo el mercado de los Plátanos y el mercado Mariscal donde se los encuentra con mayor frecuencia. Se consideró también el tipo de palma de la cual provienen los chontacuros y se encontró que la palma chonta y la palma morete son las

1 Universidad Central del Ecuador, Facultad de Ciencias Químicas, Quito Ecuador (anitaespinosa18@hotmail.com; amhidalgo@uce.edu.ec; emayorga@uce.edu.ec) 
más comunes. Para determinar la composición nutricional de los chontacuros se procedió a analizar los parámetros fisicoquímicos de humedad, ceniza, grasa y proteínas utilizando los métodos oficiales de análisis de la AOAC Internacional, mientras que, para determinar el contenido de colesterol y de sodio, se utilizaron las normas INEN. Para la identificación de los ácidos grasos se realizó la metilación de los ésteres de ácidos grasos con el método oficial AOCS Ce 2-66 y se obtuvo el perfil lipídico mediante cromatografía de gases. Como resultado se obtuvo que el valor nutricional de los chontacuros tiene un contenido del 18 \% de proteína, $35 \%$ de grasa, $1 \%$ de carbohidratos, $2 \%$ de sodio y $11 \%$ de colesterol; en cuanto a los ácidos grasos se encontró que tienen ácidos grasos insaturados del tipo omega-6 y omega-9.

\section{ABSTRACT}

The purpose of this investigation was to determine the nutritional value, as well as to identify types of fatty acids that come from chontacuro's fat of the species Rhynchophorus palmarum $L$. which were collected at the markets of the Puyo city of Pastaza province, where Plátanos and Mariscal markets have most of them. It was also considered the type of palm from which chontacuros come, it was found that the chonta and morete palm are the most common. In order to determine the nutritional composition of chontacuros, physicochemical parameters of moisture, ash, fat and proteins were analyzed using the official methods of AOAC International, while INEN standards were used to determine the cholesterol and sodium content. In the identification of fatty acids, methylation of fatty acid esters was performed with the official AOCS Ce 2-66 method and the lipid profile was obtained by gas chromatography. As a result, the nutritional value of chontacuros had $18 \%$ protein, $35 \%$ fat, $1 \%$ carbohydrate, $2 \%$ sodium and $11 \%$ cholesterol, and for fatty acids it was found to have unsaturated fatty acids such as omega 6 and 9 . 


\section{INTRODUCCIÓN}

La Organización de las Naciones Unidas para la Agricultura y Alimentación (FAO) estableció en 2013 que una de las fuentes alternativas para obtener nutrientes, sobre todo proteína, puede ser a través del consumo de insectos, los cuales han formado parte de las dietas tradicionales de al menos 2000 millones de personas.

El consumo de insectos es conocido como entomofagia y en algunos países forma parte de la cultura, entre ellos están México, Tailandia y China. Ecuador no es la excepción ya que se consumen principalmente las hormigas de limón, el catzo blanco y el chontacuro (Rhynchophorus palmarum L.), este último, conocido también como mayón, es originario de la Región Amazónica y se ha convertido en uno de los platos típicos de esta región (Poveda, 2016). Las larvas de chontacuro pertenecen al orden Coleóptera y a la familia Curculionidae (Aldana, Aldana, \& Moya, 2011).

Los chontacuros se desarrollan y alimentan en las palmas de la familia de las Arecaceae; se consideran a 11 es- pecies de palmas como su hábitat, pero con mayor frecuencia se los encuentra en la palma de la chonta (Bactris gasipaes) y la palma morete (Mauritia flexuosa), en los troncos que son cortados y que quedan en el suelo (Barragán \& Carpio, 2006).

En el Ecuador los chontacuros, además de ser un alimento para los indígenas amazónicos, son también consumidos por las personas que visitan las provincias de la Amazonía, porque son parte de la comida típica de la región. Su forma de preparación varía, pueden ser asados, cocidos, aunque también se los consume crudos. En consecuencia, es importante conocer su composición, porque en base a esta se puede obtener información sobre el valor nutricional que aportan al consumidor.

El consumo de ácidos grasos es importante en la dieta, sobre todo los ácidos grasos insaturados porque cumplen diversas funciones en el cuerpo, entre ellas que ayudan al correcto funcionamiento del sistema cardiovascular. Estos ácidos grasos se clasifican en dos grupos, los monoin- 
saturados que son ácidos grasos omega-9 y son considerados no esenciales, y los poliinsaturados que son ácidos grasos omega-3 y omega-6 y son considerados como esenciales porque el organismo no los puede sintetizar y deben ser consumidos mediante los alimentos (Ortega, 2002).

Los ácidos grasos omega-6 son importantes para el buen funcionamiento de las membranas celulares, la fuente de este tipo de ácidos grasos está en los aceites vegetales como el de soja, girasol, maíz, etc., son importantes porque una vez en el organismo se transforman en ácido araquidónico y este, a su vez, en eicosanoides, que son biomoléculas que intervienen en la regulación de la homeostasis vascular y de los procesos inflamatorios (Aranceta \& Gil, 2010).

El objetivo de la presente investigación es conocer el valor nutricional y el tipo de ácidos grasos que presentan los chontacuros, considerando el tipo de palma del que provienen y así determinar si esta influye en su composición;, además se espera que los consumidores conozcan los nutrientes y las cantidades que estosaportan en a su dieta.

\section{MATERIALES Y MÉTODOS}

Se realizó un muestreo probabilístico aleatorio simple, se seleccionaron 5 puntos de muestreo del mercado de los Plátanos y 4 puntos de muestreo del mercado Mariscal, teniendo un total de 9 puntos de muestreo. Por cada punto se recolectaron 12 unidades de chontacuros, analizándose en total 108 muestras. El análisis de cada parámetro fisicoquímico se realizó por triplicado y, para el análisis de resultados, se consideró el tipo de palma del que provenían los chontacuros.
Para los análisis de cada una de las muestras se utilizaron los métodos establecidos por la AOAC para carne y productos cárnicos, las normas INEN y el método AOCS. Para la humedad se utilizó el método AOAC 950.46 con una estufa eléctrica modelo Junior 101 a $130 \pm 3{ }^{\circ} \mathrm{C}$. Para la determinación de ceniza se utilizó el método AOAC 923.03 en una mufla eléctrica, modelo F-C 2025P.PLO. THERMOLYNE 2332 a $550{ }^{\circ} \mathrm{C}$, hasta obtener cenizas de color gris claro. 
Para el análisis de grasa se utilizó el método AOAC 960.39 con un aparato de Soxhlet de vidrio durante 4 horas. Para la determinación de proteína se utilizó el método AOAC 981.10 con un digestor de proteína marca RAYPA y un destilador de nitrógeno marca Velp Scientifica, modelo UDK127. Para la determinación de sodio se utilizó la norma NTE INEN 51: determinación de cloruro de sodio que se basa en el método de Mohr. Para colesterol se utilizó la norma NTE INEN 729, mediante el método colorimétrico en el espectrofotómetro Varian Cary 50 Bio UV-VIS a $625 \mathrm{~nm}$; y, previo al análisis de los ácidos grasos, se utilizó el método AOCS Ce 2-66. Para la preparación de metil ésteres de ácidos grasos y para su identificación se utilizó el equipo de cromatografía de gases $\mathrm{YL}$ Instrument 6500GC System, acoplado a un detector de ionización de
Ilama, con una columna capilar de fase reversa. La columna empleada fue de $60 \mathrm{~m}$ de longitud por 0,25 mm de diámetro interno SELECTA para FAME y 0,2 $\mu$ m de espesor de fase. La velocidad en la columna fue de 27,7 $\mathrm{cm} / \mathrm{s}, 2,0 \mathrm{~mL} / \mathrm{min}$ y el flujo de hidrógeno fue de $25,0 \mathrm{~mL} / \mathrm{min}$. La temperatura del inyector fue de $100{ }^{\circ} \mathrm{C}$ y la temperatura del detector fue de 260 ${ }^{\circ} \mathrm{C}$ y se utilizó como gas de arrastre nitrógeno a 300,0 mL/min.

\section{Tratamiento estadístico de datos}

Se utilizó la prueba estadística t de student en dos medias experimentales a un nivel de confianza del $95 \%$, para, de esta forma, determinar si existe diferencia significativa entre las medias de cada uno de los parámetros fisicoquímicos y de los ácidos grasos según el tipo de palma del que provienen los chontacuros.

\section{RESULTADOS}

Análisis proximal: En la Tabla 1 se muestran los resultados de cada uno de los parámetros fisicoquímicos analizados de los chontacuros de la palma morete y de la palma chonta, obteniéndose un porcentaje alto de humedad y un contenido bajo en ceniza para los chontacuros de ambas palmas. 
Tabla 1. Parámetros fisicoquímicos de los chontacuros por el tipo de palma

\begin{tabular}{lcc}
\hline & \multicolumn{2}{c}{ Chontacuros } \\
\cline { 2 - 3 } $\begin{array}{l}\text { Parámetro } \\
\text { fisicoquímico }\end{array}$ & $\begin{array}{c}\text { Palma } \\
\text { morete } \\
\text { (Mauritia } \\
\text { flexuosa) }\end{array}$ & $\begin{array}{c}\text { Palma } \\
\text { chonta } \\
\text { (Bactris } \\
\text { gasipaes) }\end{array}$ \\
\hline Humedad & $66,00 \%$ & $65,20 \%$ \\
Ceniza & $0,85 \%$ & $0,76 \%$ \\
Sodio & $50,64 \mathrm{mg}$ & $45,43 \mathrm{mg}$ \\
Proteína & $8,68 \%$ & $8,72 \%$ \\
Grasa & $23,02 \%$ & $23,61 \%$ \\
Carbohidratos & $1,44 \%$ & $1,71 \%$ \\
Colesterol & $35,05 \mathrm{mg}$ & $24,44 \mathrm{mg}$ \\
\hline
\end{tabular}

Se determinó que no hay significancia entre las medias de los parámetros fisicoquímicos, es decir, no influye el tipo de palma del que provienen los chontacuros, con estos resultados se calculó la composición promedio de los chontacuros con sus respectivas desviaciones estándar, los resultados se expresan en la Tabla 2.
Tabla 2. Composición promedio de los chontacuros

\begin{tabular}{lc}
\hline $\begin{array}{l}\text { Parámetro } \\
\text { fisicoquímico }\end{array}$ & Contenido \\
\hline Humedad & $65,82 \pm 2,26 \%$ \\
Ceniza & $0,83 \pm 0,09 \%$ \\
Sodio & $49,48 \pm 8,69 \mathrm{mg}$ \\
Proteína & $8,69 \pm 0,88 \%$ \\
Grasa & $23,15 \pm 1,50 \%$ \\
Carbohidratos & $1,50 \pm 0,94 \%$ \\
Colesterol & $32,69 \pm 9,62 \mathrm{mg}$ \\
\hline
\end{tabular}

\section{Perfil de ácidos grasos}

En la Tabla 3 se muestran los ácidos grasos identificados en la grasa de los chontacuros de la palma morete y los de la palma de la chonta en el $100 \%$ de grasa. Se determinó que existe una diferencia significativa entre los ácidos grasos encontrados en la grasa de los chontacuros, es decir, que sí influye el tipo de palma de la cual provienen. 
Tabla 3. Composición de los ácidos grasos de los chontacuros por el tipo de palma

\begin{tabular}{lcc}
\hline Tipo de ácido graso & $\begin{array}{c}\text { Chontacuros } \\
\text { palma morete } \\
(\%)\end{array}$ & $\begin{array}{c}\text { Chontacuros } \\
\text { palma chonta } \\
(\%)\end{array}$ \\
\hline (C14:0) Ácido mirístico & $3,11 \pm 0,25$ & $3,85 \pm 0,35$ \\
(C16:0) Ácido palmítico & $45,30 \pm 2,70$ & $45,45 \pm 1,06$ \\
(C16:1) Ácido palmitoleico & $1,79 \pm 0,39$ & $3,10 \pm 0,99$ \\
(C18:0) Ácido esteárico & $7,80 \pm 1,89$ & $6,40 \pm 0,57$ \\
(C18:1n-9) Ácido oleico & $38,70 \pm 3,67$ & $38,15 \pm 0,35$ \\
(C18:2n-6) Ácido linoleico & $1,83 \pm 0,52$ & $1,60 \pm 0,28$ \\
(C18:3n-6) Ácido $\gamma$-linolénico & $1,05 \pm 0,29$ & $1,05 \pm 0,21$ \\
(C20:1n-9) Ácido cis-1 1 eicosenoico & $0,78 \pm 0,13$ & 0,80 \\
Omega-6 & 2,88 & 2,65 \\
Omega-9 & 39,48 & 38,95 \\
Ácidos grasos saturados & 56,21 & 55,70 \\
Ácidos grasos monoinsaturados & 41,27 & 42,05 \\
Ácidos grasos poliinsaturados & 2,88 & 2,65 \\
\hline
\end{tabular}

Con la información de los análisis proximales y la determinación del tipo de ácidos grasos de los chontacuros por el tipo de palma del que provienen, se elaboró una tabla de información nutricional, utilizando la metodología que se indica en la norma ecuatoriana NTE INEN 1334-2. Rotulado de productos alimenticios para consumo humano. Parte 2. Rotulado nutricional. Requisitos, en la cual se establecen los requisitos que debe cumplir el rotulado nutricional de los alimentos, además de indicar los nutrientes que deben ser declarados en base a una dieta de 2000 calorías.

En la Tabla 4 se muestra la información nutricional de los chontacuros de la palma morete. . 
Tabla 4. Información nutricional de los chontacuros de la palma morete

\section{Información Nutricional}

\begin{tabular}{|c|c|}
\hline \multicolumn{2}{|l|}{ Tamaño de la porción: 100 g } \\
\hline Porciones por envase: 1 & \\
\hline \multicolumn{2}{|l|}{ Cantidad por porción } \\
\hline Energía (Calorías) & $1042 \mathrm{~kJ}(250 \mathrm{Cal})$ \\
\hline \multirow[t]{2}{*}{ Energía de grasa (Calorías de Grasa) } & $880 \mathrm{~kJ}(210 \mathrm{Cal})$ \\
\hline & \% Valor Diario* \\
\hline Grasa Total $23 \mathrm{~g}$ & $35 \%$ \\
\hline Grasa Saturada $11 \mathrm{~g}$ & $55 \%$ \\
\hline \multicolumn{2}{|l|}{ Grasa Trans $0 \mathrm{~g}$} \\
\hline \multicolumn{2}{|l|}{ Grasa Monoinsaturada $11 \mathrm{~g}$} \\
\hline \multicolumn{2}{|l|}{ Grasa Poliinsaturada $1 \mathrm{~g}$} \\
\hline Colesterol $33 \mathrm{mg}$ & $11 \%$ \\
\hline Sodio $50 \mathrm{mg}$ & $2 \%$ \\
\hline Carbohidratos Totales $2 \mathrm{~g}$ & $1 \%$ \\
\hline Proteína $9 \mathrm{~g}$ & $18 \%$ \\
\hline
\end{tabular}

*Los porcentajes de valores diarios están basados en una dieta de 2000 calorías $(8380 \mathrm{~kJ})$. Sus valores diarios pueden ser mayores o menores dependiendo de sus necesidades calóricas.

Esta es la información nutricional valor diario de grasa saturada es del para los chontacuros de la palma $65 \%$, la grasa monoinsaturada tiene chonta, el cálculo del porcentaje del un total de $9 \mathrm{~g}$.

\section{DISCUSIÓN}

En el estudio realizado por Romero y Mejía en el 2017 sobre la determinación del valor nutritivo de carne y macerado de larvas de Rhynchopho- rus palmarum L. "suri", procedentes de Moyobamba-Región de San Martín, mencionan que la composición de las larvas fue: 70,78 \% de hume- 
dad, $0,66 \%$ de ceniza, 9,34\% de proteína, $20,98 \%$ de grasa y $1,79 \%$ de carbohidratos. Al comparar estos resultados con la presente investigación los valores son cercanos para los parámetros fisicoquímicos obtenidos, aunque el porcentaje de humedad, grasa y proteína presentaron mayor porcentaje en la composición de los chontacuros.

La diferencia del contenido de humedad para los chontacuros de la palma morete y los chontacuros de la palma chonta puede estar relacionada con la fuente de alimentación y las condiciones ambientales.

La ceniza representa el contenido total de minerales en los alimentos (Nielsen, 2003). Los chontacuros presentaron $0,83 \%$ de ceniza, contenido que es bajo.

La grasa en los chontacuros fue de $23,15 \%$. Los insectos que se encuentran en la etapa de larva acumulan mayor cantidad de grasa y la utilizarán durante la metamorfosis para convertirse en escarabajos (Poveda, 2016).

La publicación de la FAO en el 2013 sobre insectos comestibles, menciona que el contenido de proteína varía en los insectos según la etapa de metamorfosis en la que se encuentren y que los adultos son los que tienen mayor contenido de proteína; también indica que las larvas de Rhynchophorus palmarum tienen entre 7-36 \% de proteína. Los chontacuros, al estar en la etapa de larva contienen menor contenido de proteína, cuyo valor fue de $8,69 \%$, el mismo que está dentro de lo que menciona la FAO en su publicación.

Para los ácidos grasos, la diferencia significativa que se dio en el ácido mirístico y el ácido palmitoleico, puede deberse a la composición de los aceites de cada una de las palmas, lo cual influye, a su vez, en la composición de los ácidos grasos de los chontacuros.

En el estudio sobre el perfil de ácidos grasos en la pulpa de aguaje, moriche o morete (Mauritia flexuosa) desarrollado por Restrepo, et al., (2016), se menciona que el aceite de morete tiene 0,06 \% de ácido mirístico y 0,29 \% de ácido palmitoleico. Para la palma de la chonta, en el estudio sobre la caracterización del extracto 
lipídico del aceite de 4 variedades de chontaduro (Bactris gasipaes), realizado por Restrepo, et al., (2012), se indica que el aceite extraído de chonta o chontaduro presenta $0,12 \%$ de ácido mirístico y 7,9 \% de ácido palmitoleico. Con estas referencias, se determina que los ácidos grasos mirístico y palmitoleico se encuentran en mayor proporción en el aceite de chonta (Bactris gasipaes), lo cual se compara con el porcentaje de los ácidos grasos identificados en la presente investigación (Tabla 3), ya que también los chontacuros de la palma chonta presentaron valores altos de estos ácidos grasos en comparación con los chontacuros de la palma morete.

En el estudio realizado por Landívar (2012), sobre la evaluación del método de digestión alcalina para la extracción de grasa de larvas de Rhynchophorus palmarum L., se identificaron ácidos grasos como el laúrico, mirístico, palmítico, palmitoleico, esteárico, oleico, linoleico y linolénico. Al compararlos con los ácidos grasos de los chontacuros de la palma morete y los chontacuros de la palma chonta, se observa que se obtienen la mayoría de los ácidos grasos enumerados con excepción del ácido cis-11-eicosenoico, encontrado en la presente investigación, y el ácido laúrico, encontrado en el estudio mencionado.

En la Tabla 3 se muestra que los ácidos grasos en mayor porcentaje de los chontacuros, tanto de la palma morete como de la palma chonta, son el ácido palmítico $(45,30 \%$ y $45,45 \%)$ y el ácido oleico (38,70 \% y $38,15 \%$ ), en contraste con el estudio de Landívar (2012), quien reporta que los ácidos grasos más representativos de la grasa de los chontacuros, son el ácido palmítico y el ácido oleico, siendo este último el de mayor porcentaje $(59,20 \%)$.

A partir de la composición nutricional de los chontacuros, tanto de la palma morete como de la palma chonta, se puede establecer que el consumo de $100 \mathrm{~g}$ de chontacuros tienen un valor energético de 250 Cal, un aporte nutricional del $18 \%$ de proteína, un $55 \%$ de grasa saturada en chontacuros palma morete $y$ un $65 \%$ en chontacuros palma chonta. 


\section{CONCLUSIONES}

Como valor nutricional de los chontacuros, uno de los sus aportes principales es el de la proteína, además son una fuente moderada de colesterol, son bajos en sodio y carbohidratos.

Los chontacuros se caracterizan por el contenido de grasa que presentan, con el análisis proximal realizado se determinó que tienen un 23,15\% de grasa.
Como ácidos grasos se identificaron tanto ácidos grasos saturados como insaturados, dentro de éstos últimos se destacan los omegas 6, considerados como ácidos grasos esenciales porque el organismo no los puede sintetizar y se los debe consumir a través de los alimentos.

\section{LISTA DE REFERENCIAS}

Aldana, R., Aldana, J., \& Moya, O. (2011). Manejo del picudo Rhynchophorus palmarum L. (Coleóptera: Curculionidae). Obtenido de Instituto Colombiano Agropecuario: https://www.ica.gov.co/getattachment/19e016c0-0d14-4412-af1203eecfe398f2/Manejo-del-picudo—Rhynchophorus-palmarum-L_(Cole.aspx

Aranceta, J., \& Gil, Á. (2010). En Alimentos funcionales y salud en la etapa infantil (pág. 6). Madrid: Médica Panamericana.

Barragán, Á., \& Carpio, C. (2006). Plantas como alimento de invertebrádos útiles. Enciclopedia de las plantas, 76-79.

FAO. (2013). Insectos comestibles: perspectivas futuras para la seguridad alimentaria . Obtenido de Organización de las Naciones Unidas para la Alimentación y Agricultura: http://www.fao.org/3/i3253e/i3253e.pdf 
Landívar, M. (2012). Evaluación del método de digestión alcalina para la extracción de grasa de larvas de Rhynchophorus palmarum L.". Puyo: Universidad Estatal Amazónica. Obtenido de https://repositorio.uea.edu.ec/bitstream/123456789/22/1/TE SIS\%20DE\%20MARCOS\%20DAVID\%20LAND\%c3\%8dVAR\%20VALVERDE.pdf

Nielsen, S. (2003). En Análisis de los alimentos (págs. 99-176). España: Acribia.

Ortega, R. (2002). Importancia de las grasas en la alimentación. Nutrición clínica y dietética hospitalaria , 4-8. Obtenido de https://revista.nutricion.org/hemeroteca/revista_agosto_03/Funcionales/FLORAgrasas.pdf

Poveda, J. (2016). Insectos y alimentación. Mundo Investigación, 2(1), 168.

Restrepo, J., Arias, N., \& Madriñan, C. (2016). Determinación del valor nutricional, perfil de ácidos grasos y capacidad antioxidante de la pulpa de aguaje (Mauritia flexuosa). Revista de Ciencias, 20(1), 71-78. Obtenido de http://revistas.univalle. edu.co/index.php/revista_de_ciencias/article/view/6109/8321

Restrepo, J., Vinasco, E., \& Estupiñan, J. (2012). Estudio comparativo del contenido de ácidos grasos en 4 variedades de chontaduro (Bactris gasipaes) de la región del pacífico colombiano. Revista de Ciencias, 126. Obtenido de http://revistas.univaIle.edu.co/index.php/revista_de_ciencias/article/view/508/630

Romero, E., \& Mejía, V. (2017). Determinación del valor nutritivo de carne y macerado de larvas de Rhynchophorus palmarum L. "suri", procedentes de Moyobamba-Región de San Martín. Moyobamba: Universidad Privada Antonio Guillermo Urrelo. 